\section{Parry-Romberg syndrome: A case report of non-invasive treatment}

\section{Anahita Dehghani Soltani ${ }^{1}$, Azita Tehranchi ${ }^{2}$, Shiva Safari ${ }^{3}$, Zahra Tehrani $^{3}$ and Samin Ghaffari ${ }^{4 *}$}

\author{
${ }^{1}$ Postgraduate Student of Orthodontics, Dental Research Center, Research Institute of Dental \\ Sciences, Dental School, Shahid Beheshti University of Medical Sciences, Tehran, Iran \\ ${ }^{2}$ Professor and Chairwomen, Department of Orthodontics, Dental Research Center, Research \\ Institute of Dental Sciences, Dental School, Shahid Beheshti University of Medical Sciences, \\ Tehran, Iran \\ ${ }^{3}$ Orthodontist, Private Practice, Tehran, Iran \\ ${ }^{4}$ Postgraduate Student of Orthodontics, Dentofacial Deformities Research Center, Research Institute \\ of Dental Sciences, Dental School, Shahid Beheshti University of Medical Sciences, Tehran, Iran
}

\section{Abstract}

Parry-Romberg syndrome is an uncommon condition, self-limiting with slow progressive hemifacial atrophy. This syndrome can lead to several progressive congenital and developmental deformities. It can cause severe facial asymmetry and subsequently lead to esthetic and psychological problems and adversely affect patient's quality of life, so its treatment holds great importance. Still, there is no exact treatment protocol for this disease, treatment approaches are bounded and patient's response to the treatment is imponderable. However, most of the patients can benefit from conservative treatments. In this paper, we have reported a moderate case of Parry-Romberg syndrome, with no familial history of any syndromes. We also have discussed about present anomalies and the steps of exerted conservative treatments.

\author{
More Information \\ *Address for Correspondence: Samin Ghaffari, \\ Postgraduate Student of Orthodontics, \\ Dentofacial Deformities Research Center, \\ Research Institute of Dental Sciences, Dental \\ School, Shahid Beheshti University of Medical \\ Sciences, Tehran, Iran, Tel: +989141077341; \\ Email: saminghaffari@gmail.com
}

Submitted: 23 December 2019 Approved: 27 December 2019 Published: 30 December 2019

How to cite this article: Soltani AD, Tehranchi A, Safari S, Tehrani Z, Ghaffari S. Parry-Romberg syndrome: A case report of non-invasive treatment. J Oral Health Craniofac Sci. 2019; 4: 015-020.

\section{DOI: 10.29328/journal.johcs.1001029}

Copyright: (c) 2019 Soltani AD, et al. This is an open access article distributed under the Creative Commons Attribution License, which permits unrestricted use, distribution, and reproduction in any medium, provided the original work is properly cited.

Keywords: Parry Romberg; Hemifacial Atrophy Syndrome; Conservative treatment; Case report

\section{(W) Check for updates}

OPEN ACCESS

\section{Introduction}

Parry-Romberg, first introduced by Parry in 1825 and named as a syndrome by Romberg in 1846, is a rare slowly progressive and self-limiting disease which leads to unilateral facial atrophy [1-4]. This syndrome is more prevalent in women and its etiology is relatively unknown $[5,6]$. Different etiologic factors have been proposed such as viral infections, genetics, trauma, peripheral and trigeminal neuritis, localized scleroderma and endocrine disorders, although, none have been substantiated [7-9]. Some researchers consider this syndrome as a spectrum of linear scleroderma [10]. In some cases, its signs can be detected at birth, in some others from 10 years old and in a few cases after 25 years old [6]. The earlier the signs of the disease start, the more severe the deformity, because of the high velocity of growth at these ages [11]. With age, the atrophy outspreads to the bottoms layers of muscles, bones and cartilage [12]. In 50 percent of the patients neurologic involvement can be seen such as neuropathic pain, epilepsy and intracerebral dysplasia [13].

This syndrome is diagnosed on the basis of clinical symptoms. In clinical examinations, small primary lesions can be seen above the eyebrows and in the frontal paramedian area, which becomes more atrophic during the progression of disease and separates the border of the healthy and atrophic tissues, called en coup de sabre $[10,14,15]$. Also, deforming facial asymmetry can be seen, effecting the oral functions due to involvement of masticatory muscles $[11,16]$. Sometimes, it accompanies with local vitiligo, hyperpigmentation or port wine stains. Also, ears can be deformed or smaller than normal $[17,18]$. Lower one third of the face including mandible and chin and also tip of nose are deviated to the affected side. Deviation of the lips causes increasing of tooth show on the affected side relative to the opposite side $[11,19]$.

Intraoral findings includes hemi-atrophy of lips, tongue and soft palate on the affected side. Also, malformation and congenital missing of teeth, delayed eruption and underdevelopment or resorption of roots can be detected [20-22]. Alveolar ridge, body of mandible, ramus and gonial angle can be smaller and underdeveloped on the affected side, which leads to deviation of dental and skeletal midlines $[23,24]$. This syndrome can cause psychological effects, disrupting the patient's daily functioning and negatively affecting patient's appearance. So, the treatment of this 
disease requires multidisciplinary approach and specified treatment planning for each patient [10]. Most of the patients can benefit from conservative treatments. Treatments vary from prescribing local or systemic anti-inflammatory drugs to reconstructive and orthognathic surgeries $[10,25]$. In this paper, we have reported a moderate case of Parry-Romberg syndrome, with no familial history of any syndromes. We have discussed about present anomalies and the steps of exerted conservative treatments. Written informed consent has been taken from the patient to use her medical records and photographs for this report. This case report is compatible with the CARE criteria [26].

\section{Results}

An 11-year-old Iranian girl was referred to orthodontic department of Shahid Beheshti dental school by her family dentist due to dental anomalies. Patient's mother pointed that she has noticed facial asymmetry and deformity of the right side of her daughter's face from 10 years old. Patient had no familial history of specific diseases or syndromes. Also, patient had no learning difficulties. In medical examinations, including cardiac evaluation via ECG, ENT consulting, assessment of internal organs via ultrasound imaging, autoimmune diseases and gene mutations by taking blood samples, no disorders were identified. In MRI imaging of brain without contrast in T1 and T2, abnormal signaling at the depth of periventricular white matter and multiple small hotspots of low signaling at the depth of white matter, thalamus and head of caudate were observed in the right side, which represent a possible previous infraction. Although, the patient stated no history of neuropathic pain, migraine or epilepsy.

In initial extraoral examination, facial asymmetry with mild depression of paranasal and frontal paramedian tissues at the right side, deviation of nose tip and chin point to the affected side and port wine stains on the right gonial area of mandible were observed. Ears, eyes, eyebrows and scalp were normal and masticatory muscles were not involved. Also, lips were inclined upwards to the right, causing asymmetry in patient's smile and tooth show at rest and while smiling (Figure $1 \mathrm{a}$-d). In intraoral examination, $\frac{621 \mid}{61 \mid}$ were hypoplastic and had smaller size in comparison to the opposite side. The lower right central incisor was severely hypoplastic. Maxillary arch was asymmetric within collapsed right side which caused right anterior and posterior crossbite. Tongue and gingival tissue were normal. There was a transverse cant in the maxillary occlusal plane to the right side and maxillary arch had yaw rotation to the right (Figure $1 \mathrm{e}-\mathrm{h}$ ).

In radiographic examination (Figure 1i,j), lower right central incisor had very short root, which caused the patient to lose this tooth at age 12.621 had coronal pulp stone and $\overline{3 \mid 13}$ had dilaceration in the middle third of their root. Upper

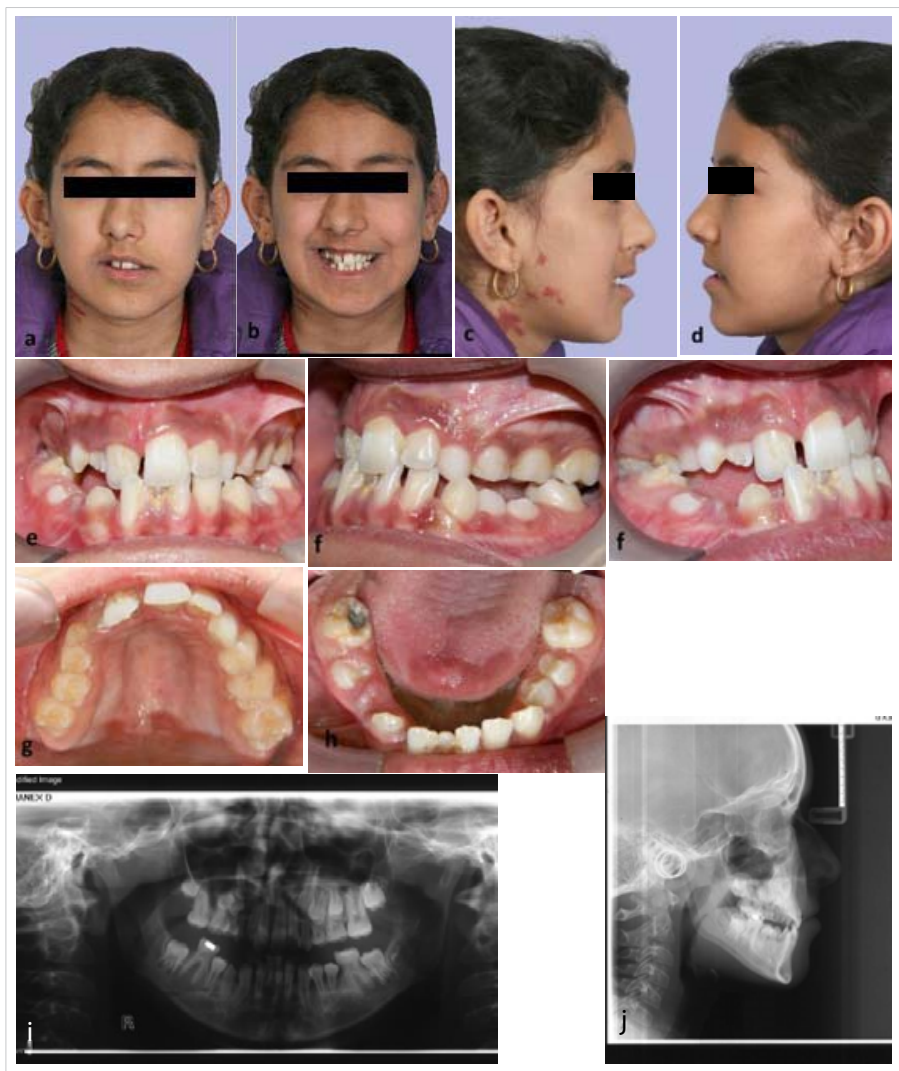

Figure 1: Patient at age 11, (a-d) extra-oral photographs, (e-h) intraoral photographs, (i) panoramic radiography, (j) lateral cephalometric radiography.

right premolar teeth's root was not developed, although half of the upper left premolar teeth's root was developed. Upper right canine was unerupted, had smaller size and the root apex was closed despite the lack of eruption, which prognosticates the possibility of impaction. In panoramic radiography, the height of mandibular ramus was similar on both sides but the condyles was different in shape. In lateral cephalometric radiography, upper and lower jaw had mild deficiency. After consultation with oral and maxillofacial surgeon, the final decision was to exert conservative treatment for the patient and to wait until the completion of growth and stabilization of the severity of facial asymmetry and then reevaluate for orthognathic surgery.

In the first phase of orthodontic treatment, Schwartz removable plate with expansion screw was prescribed to correct constriction of maxillary arch. With good patient cooperation, overcorrection of crossbite reached after 6 months and then plate was used as a retainer for 6 months. Before starting the second phase of orthodontic treatment at age 13, the patient was re-examined (Figure 2). In extraoral examination, nasolabial sulcus was deepened and the facial asymmetry and deviation of nose, chin and mouth were mildly intensified. In intraoral examination, the lower right central incisor was lost, as mentioned before due to severely short root. The upper right canine and second premolar teeth were impacted, which was also confirmed by CBCT (Figure 3). The upper right primary canine had no mobility. The upper right first premolar was starting to erupt. So, the present teeth were: 


\section{$64 C 21 \mid 123456$}

$\overline{765432 \mid 123456}$

In order to obtain proper functional occlusion and better esthetics, fixed orthodontic treatment was started at age 13 . Roth 0.022 inch slot brackets were bonded on all present teeth and leveling and aligning was conducted by light forces due to short roots (Figure 4). Midline of upper and lower arches were corrected and coordinated with facial midline. Due to grade 2 mobility of hypoplastic teeth and dilaceration of roots, optimal root paralleling was not obtained. Considering multiple factors, such as no mobility of upper right primary canine, extremely short root of upper right second premolar, impossibility of providing proper anchorage due to mobility of adjacent teeth and patient's age, and also up on patient's own decision, it was decided to do no intervention for the impacted teeth. The active orthodontic treatment time was 20 months. In comparison to the previous radiographs and photographs, the asymmetry was not significantly increased. After removal of orthodontic appliances, the patient was referred to cosmetic and restorative dentistry department for composite buildup of upper right anterior teeth and composite veneer of upper and lower right first premolars. Removable upper and lower Hawley plates were prescribed for retention. Eventually, patient's satisfaction with the treatment was very high (Figure 5).

\section{Discussion}

Parry Romberg syndrome can lead to several progressive congenital and developmental deformities [27]. It leads to unilateral atrophy of facial muscles, bones and skin but typically and mainly fat. It is more prevalent in female population and usually, the atrophy is restricted to the one side of the face. Also, the skin of the affected side can be dry and can have hyperpigmentation such as port wine stains [28], that all of them were observed in the presented case.

This syndrome can be classified according to its severity [10]:

- Mild Parry Romberg syndrome: Atrophy of soft tissues at the trigeminal area of lower face without rotation of the occlusal plane.

- Moderate Parry Romberg syndrome: Involvement of nostrils and lip commissures in addition to the trigeminal dermatome, no atrophy of skeleton and relatively horizontal occlusal plane.

- Severe Parry Romberg: Rotation and transverse cant of occlusal plane, atrophy of maxilla, mandible and zygoma, deviation of chin and nose to the affected side.

In this case, considering the mild atrophy of skeleton, mild rotation and transverse cant of occlusal plane which was mostly due to small size of upper and lower right teeth and moderate facial asymmetry, it can be considered as

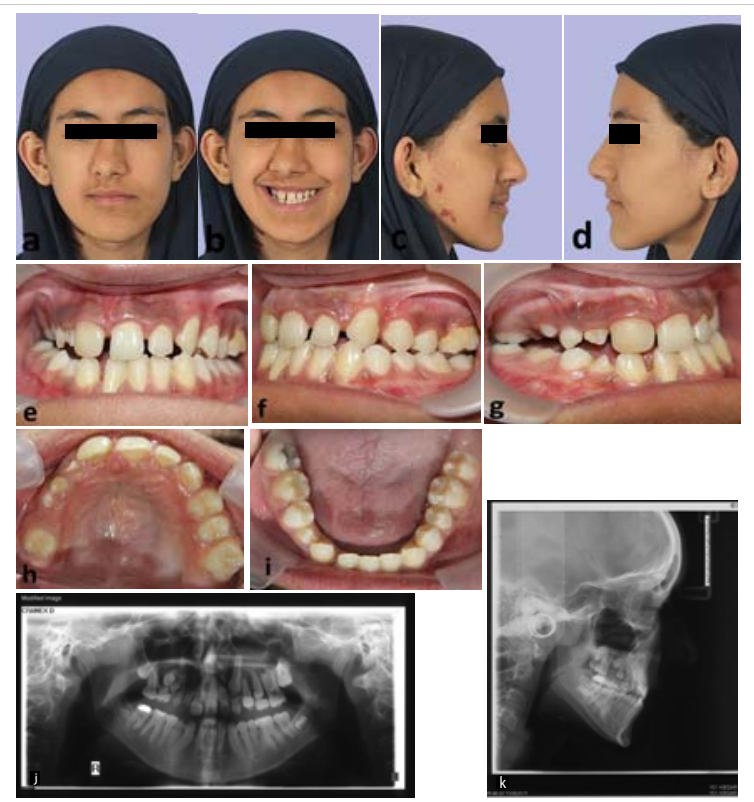

Figure 2: Patient at age 13, (a-d) extra-oral photographs, (e-i) intraoral photographs, (j) panoramic radiography, (k) lateral cephalometric radiography.
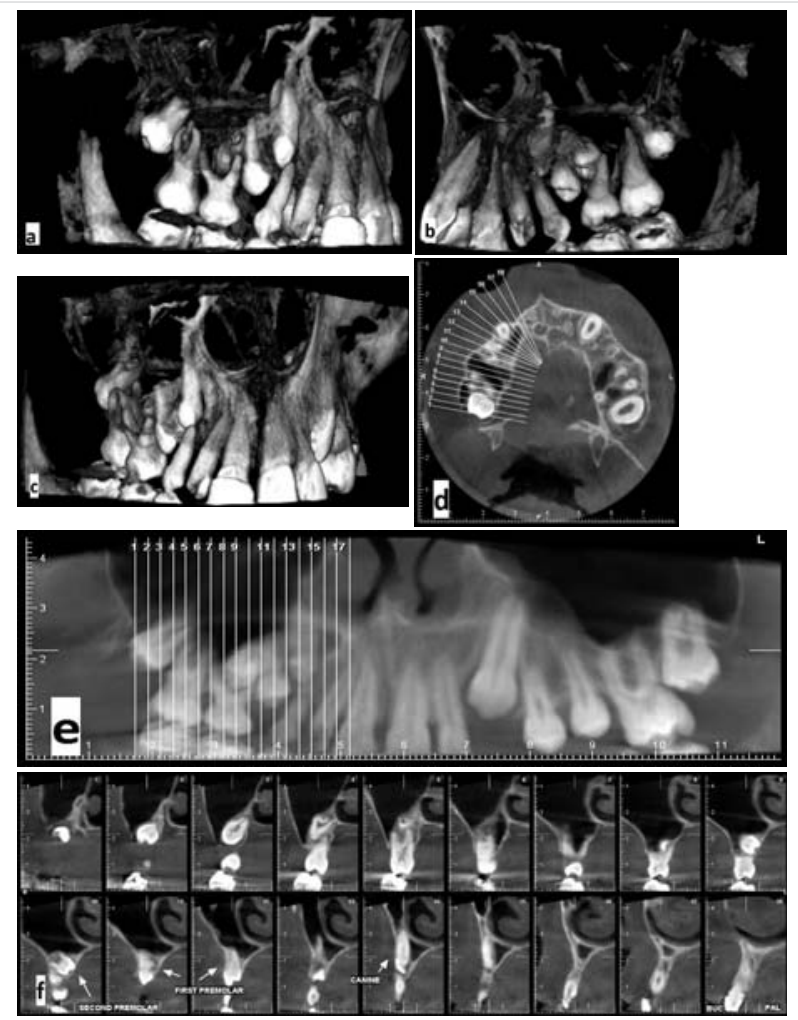

Figure 3: CBCT radiographs at age 13, (a) buccal view, (b) palatal view, (c) anterior view, (d) sagittal view, (e) panoramic view of upper arch, (f) sections of impacted teeth with slice distance of $2 \mathrm{mms}$.

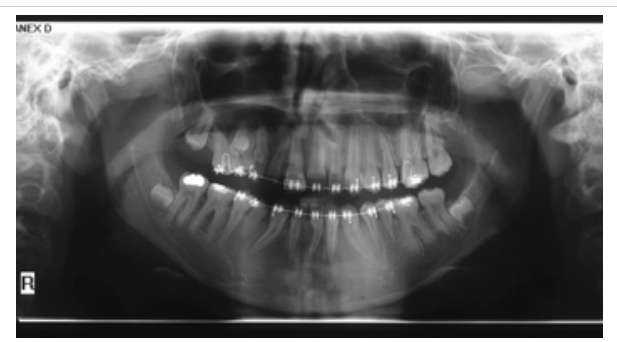

Figure 4: Panoramic radiograph at age 14 , during orthodontic treatment. 


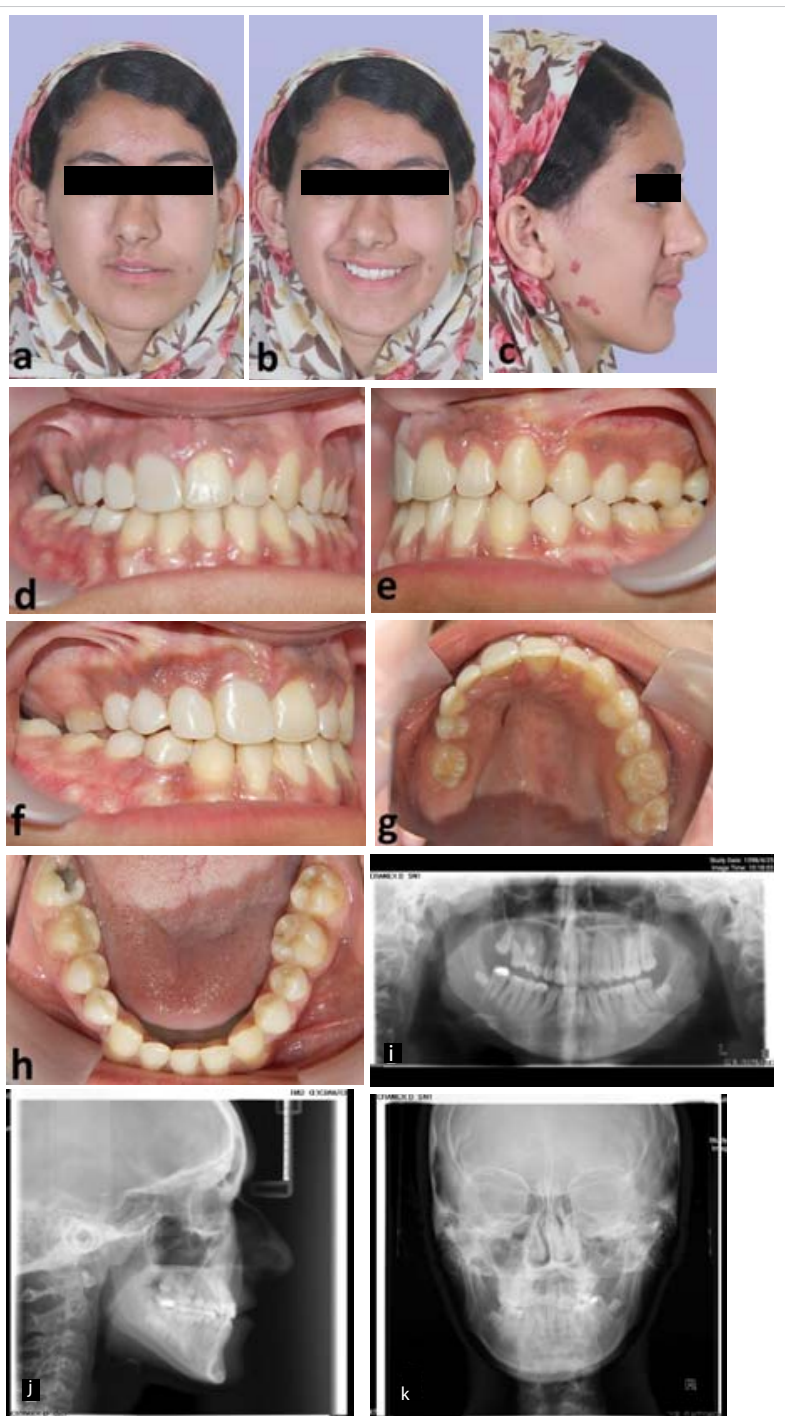

Figure 5: Patient at age 15, (a-c) extra-oral photographs, (d-h) intraora photographs, (i) panoramic radiography, (j) lateral cephalometric radiography, (k) PA cephalometric radiography.

a moderate Parry Romberg syndrome. Also after growth spurt, the asymmetry mildly increased but the rate of progression was very low. Mazzeo, et al. first reported this syndrome with root atrophy of maxillary and mandibular teeth and pulp stones in first permanent molars [16]. This patients had the mentioned manifestations and also other various manifestations reported in previous literatures $[3,5,10,11,15,16,25,27]$. On the affected side, teeth was hypoplastic and had normal morphology but with smaller size. Also some of the teeth had dilaceration of root. Also, enamel hypoplasia, pulp stones, short roots and delay in eruption was detected on the affected side.

It has been proven that, mesenchymal structures of head and neck, including facial dermis, muscles and skeleton, are originated from neural crests. Neural crests are created from distal neural tube and can be differentiated to various cells $[29,30]$. Also, neural crests are requisite for morphogenesis of teeth and formation of dentin, pulp, cementum and periodontal ligament [31]. So, the formation and function of neural crest cells can play a role as an etiologic factor for Parry Romberg syndrome [32]. As mentioned, this syndrome can cause severe facial deformity and subsequently lead to esthetic and psychological problems and adversely affect patient's quality of life [33]. Also, it is self-restrictive and the symptoms usually stabilize 5 to 10 years after observing the first symptoms [34]. However, there is no exact treatment protocol for this disease, treatment approaches are bounded and patient's response to the treatment is imponderable [35]. So, most of the patients benefit from symptomatic treatment to control neurologic signs such as neurologic pains or seizures, maxillofacial surgery after stabilization of facial deformity and immunosuppressive therapies by multidisciplinary attendance of neurologists, dentists and psychologists $[36,37]$. Cosmetic treatments, which is a major concern for most of the patients, can be exerted after growth spurt and cessation of disease's evolution and includes augmentation of atrophic side, orthognathic surgery for correction of asymmetry, silicone injections and prostheses [38]. In this patient, considering the moderate severity of disease and incomplete growth, which predispose to further changes after growth spurt, we decided to treat patient with conservative approaches and make the final decision for orthognathic surgery after completion of growth. In the first phase at age 11, patient used removable Schwartz plate to expand constricted maxillary arch. Then in the second phase at age 13, comprehensive orthodontic treatment was started in order to obtain functional occlusion and better esthetics. Multiples factors caused to compromise the treatment of impacted teeth. Active orthodontic treatment lasted 20 months and after completion of treatment, composite buildups and composite veneers were applied for hypoplastic teeth and Hawley retainers were prescribed to maintain obtained results. At the end of treatment, patient and her parents were pleased with the results.

During orthodontic treatment, patient had no neurologic problems such as seizure, migraine or neurologic pains. According to previous blood tests, autoimmune disease had no role on this syndrome's occurrence. So, the patient did not receive any immunosuppressive therapy. At the end of treatment, that patient was 15 years old, she was re-referred to oral and maxillofacial surgeon for consultation on cosmetic surgery. For correction of soft tissue asymmetry, autogenous fat graft was suggested. Autogenous fat graft, based on adipose tissue repositioning, is one of the safe and less invasive treatments for atrophy and asymmetry of mild to moderate Parry Romberg syndrome [39]. Other alternatives are cartilage grafts, inorganic implants, silicone injections and bovine collagen [28]. A retrospective observational study conducted by Amaral, et al. evaluated 14 treated patients and concluded that satisfactory results were obtained by different surgical approaches upon the severity of the deformity. Also they exhibited that the severity of deformity is not correlated with quality of life of affected patients [40]. The patient was currently reluctant to undergo surgery. 
In summary, Parry Romberg syndrome is an uncommon condition, self-limiting with slow progressive hemifacial atrophy. Its exact etiology and pathophysiology are still unknown. This syndrome causes esthetic, functional and psychological issues, which require a well-planned, specific and multidisciplinary treatment. It this paper, we presented a case of Parry Romberg syndrome with classical features. We used a non-invasive approach with comprehensive orthodontic treatment and a final restorative treatment. We succeeded to reach good results with high satisfaction of the patient. The patient was recommended for autogenous fat graft for soft tissue asymmetry in the future. So, this case is a proper sample of patients, who can benefit from noninvasive treatments.

\section{References}

1. Parry $\mathrm{CH}$. Collections from the Unpublished Medical Writings of the Late Caleb Hillier Parry. Med Chir Rev. 1825; 2. 124-147.

PubMed: https://www.ncbi.nlm.nih.gov/pubmed/29917749

2. Moore T, Southard KA, Casko JS, Qian F, Southard TE. Buccal corridors and smile esthetics. American Journal of Orthodontics and Dentofacial Orthopedics. 2005; 127: 208-213.

PubMed: https://www.ncbi.nlm.nih.gov/pubmed/15750540

3. Al-Khenaizan S, Al-Watban L. Parry-Romberg syndrome: Overlap with linear morphea. Saudi medical journal. 2005; 26: 317-319.

PubMed: https://www.ncbi.nlm.nih.gov/pubmed/15770315

4. Hickman JW, Sheils WS. Progressive facial hemiatrophy: report of a case with marked homolateral involvement. Arch Intern Med. 1964; 113: $716-720$.

PubMed: https://www.ncbi.nlm.nih.gov/pubmed/14120599

5. Miller $M$, et al. Progressive hemifacial atrophy (Parry-Romberg disease). Journal of pediatric ophthalmology and strabismus. 1987; 24: 27-36.

6. Tolkachjov SN, Patel NG, Tollefson MM. Progressive hemifacial atrophy: A review. Orphanet J Rare Dis. 2015; 10: 39.

PubMed: https://www.ncbi.nlm.nih.gov/pubmed/25881068

7. Wolf SM. Verity, Neurological complications of progressive facial hemiatrophy. Journal of Neurology, Neurosurgery \& Psychiatry. 1974: 37: 997-1004.

8. Asher SW. Berg BO. Progressive hemifacial atrophy: report of three cases, including one observed over 43 years, and computed tomographic findings. Arch Neurol. 1982: 39: 44-46.

PubMed: https://www.ncbi.nlm.nih.gov/pubmed/7055447

9. Moss $M$, Crikelair G. Progressive facial hemiatrophy following cervical sympathectomy in the rat. Arch Oral Biol. 1960; 1: 254-314.

PubMed: https://www.ncbi.nlm.nih.gov/pubmed/14424619

10. Van der Cruyssen F, Van der Cruyssen F, Schoenaersb MJ, Politis C. Parry Romberg syndrome: A long-term retrospective cohort study of 10 patients. Oral and Maxillofacial Surgery Cases. 2018; 4: 73-83.

11. O'Flynn S, Kinirons M. Parry-Romberg syndrome: a report of the dental findings in a child followed up for 9 years. Int $\mathrm{J}$ Paediatr Dent. 2006; 16: 297-301.

PubMed: https://www.ncbi.nlm.nih.gov/pubmed/16759329

12. Zafarulla M. Progressive hemifacial atrophy: a case report. British journal of ophthalmology. 1985; 69: 545-547.

13. Moko SB, Mistry Y, De Chalain TMB. Parry-Romberg syndrome: intracranial MRI appearances. J Craniomaxillofac Surg. 2003; 31: 321-324. PubMed: https://www.ncbi.nlm.nih.gov/pubmed/14563334

14. Taylor HM, Robinson R, Cox T. Progressive facial hemiatrophy: MRI appearances. Dev Med Child Neurol. 1997; 39: 484-486. PubMed: https://www.ncbi.nlm.nih.gov/pubmed/9285440

15. Rischebieth R. Progressive facial hemiatrophy (Parry-Romberg syndrome). Proceedings of the Australian Association of Neurologists. 1976; 13: 109-112.

16. Mazzeo N, et al. Progressive hemifacial atrophy (Parry-Romberg syndrome) case report. Oral Surg Oral Med Oral Pathol Oral Radiol Endod. 1995; 79: 30-35.

PubMed: https://www.ncbi.nlm.nih.gov/pubmed/7614157

17. Menni S, Marzano AV, Passoni E. Neurologic abnormalities in two patients with facial hemiatrophy and sclerosis coexisting with morphea. Pediatr Dermatol. 1997; 14: 113-116.

PubMed: https://www.ncbi.nlm.nih.gov/pubmed/9144696

18. Finesilver B, Rosow HM. Total hemiatrophy. J Am Medical Association. 1938; 110: 366-368.

19. Miller M, Spencer M. Progressive hemifacial atrophy. A natural history study. Trans Am Ophthalmol Soc. 1995; 93: 203.

PubMed: https://www.ncbi.nlm.nih.gov/pubmed/8719679

20. Rees TD, Ashley FL, Delgado JP. Silicone fluid injections for facial atrophy. Plast Reconstr Surg. 1973; 52: 118-127.

PubMed: https://www.ncbi.nlm.nih.gov/pubmed/4578999

21. Colquhoun A, Ferguson M, Doyle T. Hemifacial atrophy with bilateral short roots. Br J Oral Maxillofac Surg. 2000; 38: 533-536. PubMed: https://www.ncbi.nlm.nih.gov/pubmed/11010789

22. Foster $\mathrm{T}$. The effects of hemifacial atrophy on dental growth. Br Dent J. 1979; 146: 148-150.

PubMed: https://www.ncbi.nlm.nih.gov/pubmed/283809

23. Bramley $\mathrm{P}$, Forbes $\mathrm{A}$. A case of progressive hemiatrophy presenting with spontaneous fractures of the lower jaw. Br Med J. 1960; 1: 1476. PubMed: https://www.ncbi.nlm.nih.gov/pubmed/13803822

24. Glass D. Hemifacial atrophy. Br J Oral Surg. 1963; 1: 194-199. PubMed: https://www.ncbi.nlm.nih.gov/pubmed/14122419

25. Jun JH. Parry-Romberg syndrome with en coup de sabre. Annals of dermatology. 2011; 23: 342-347.

26. Gagnier JJ. The CARE guidelines: consensus-based clinical case reporting guideline development. Glob Adv Health Med. 2013; 7: 223. PubMed: https://www.ncbi.nlm.nih.gov/pubmed/24416692

27. Tang XJ. Parry-Romberg syndrome with rare maxillofacial deformities: A report on two cases. J Craniomaxillofac Surg. 2014; 42: 780-783.

PubMed: https://www.ncbi.nlm.nih.gov/pubmed/24359863

28. Rangare AL. Parry-Romberg syndrome: a rare case report. J Oral Maxillofac Res. 2011; 2.

PubMed: https://www.ncbi.nlm.nih.gov/pubmed/24421992

29. Noden DM. The role of the neural crest in patterning of avian cranial skeletal, connective, and muscle tissues. Dev Biol. 1983; 96: 144-165. PubMed: https://www.ncbi.nlm.nih.gov/pubmed/6825950

30. Bronner-Fraser M. Mechanisms of neural crest cell migration. Bioessays. 1993; 15: 221-230.

31. Chai Y. Fate of the mammalian cranial neural crest during tooth and mandibular morphogenesis. Development. 2000; 127: 1671-1679. PubMed: https://www.ncbi.nlm.nih.gov/pubmed/10725243 
32. Pichiecchio A. Parry-Romberg syndrome with migraine and intracranial aneurysm. Neurology. 2002; 59: 606-608.

PubMed: https://www.ncbi.nlm.nih.gov/pubmed/12196658

33. Onesti MG. Minimally invasive combined treatment for Parry-Romberg syndrome. Aesthetic plastic surgery. 2009; 33: 452-456.

PubMed: https://www.ncbi.nlm.nih.gov/pubmed/19093144

34. Stone J. Parry-Romberg syndrome. Practical Neurology. 2006; 6: 185188.

35. Wong M. Parry Romberg syndrome: 7 cases and literature review. AJNR Am J Neuroradiol. 2015; 36: 1355-1361.

PubMed: https://www.ncbi.nlm.nih.gov/pubmed/26066627

36. KorkmazC,AdapinarB,UysalS. Beneficial effect of immunosuppressive drugs on Parry-Romberg syndrome: a case report and review of the literature. South Med J. 2005; 98: 940-943.

PubMed: https://www.ncbi.nlm.nih.gov/pubmed/16217992
37. De Vasconcelos Carvalho M. Association of aesthetic and orthodontic treatment in Parry-Romberg syndrome. J Craniofac Surg. 2010; 21: 436-439.

PubMed: https://www.ncbi.nlm.nih.gov/pubmed/20216455

38. Roddi R. Clinical evaluation of techniques used in the surgical treatment of progressive hemifacial atrophy. J Craniomaxillofac Surg. 1994; 22: 23-32.

PubMed: https://www.ncbi.nlm.nih.gov/pubmed/8175994

39. Rodby KA. Evaluating Autologous Lipofilling for Parry-Romberg Syndrome-Associated Defects: A Systematic Literature Review and Case Report. Cleft Palate Craniofac J. 2016; 53: 339-350. PubMed: https://www.ncbi.nlm.nih.gov/pubmed/26295800

40. Raposo-Amaral CE. Parry-Romberg syndrome: severity of the deformity does not correlate with quality of life. Aesthetic Plast Surg. 2013; 37: 792-801.

PubMed: https://www.ncbi.nlm.nih.gov/pubmed/23720075 